\title{
Humidity Compensation by Neural Network for Bad-Smell Sensing System Using Gas Detector Tube and Built-in Camera
}

\author{
Takamichi Nakamoto*, Tomohiro Ikeda, \\ Hiroyuki Hirano and Takemi Arimoto ${ }^{1}$ \\ Department of Physical Electronics, Graduate School of Science and Engineering, \\ Tokyo Institute of Technology, 2-12-1 Ookayama, Meguro-ku, Tokyo 152-8552, Japan \\ ${ }^{1}$ Gastec Corporation, 8-8-6 Fukayanaka, Ayase, Kanagawa 252-1195, Japan
}

(Received May 31, 2010; accepted August 30, 2010)

Key words: gas detector tube, humidity, neural network, bad smell, FPGA

A cheap and rapid sensing system is required for detecting bad smells and volatile organic compounds (VOCs). Although detection using a gas detector tube is a simple gas detection method, its measurement process has not been automated. We studied an automated measurement system for gas detector tubes using a built-in camera. Although the measurement was automated using our system, another problem was revealed. Because digital cameras are sensitive to color changes, a slight change due to humidity, which is not a problem in manual inspections, cannot be ignored in our system. Thus, a humidity sensor was added to the system. However, simple humidity compensation methods such as linear regression did not work because the humidity affected the data in a complicated manner. Therefore, a neural network was used for humidity compensation. Both the discoloration area and humidity data were input to the neural network. As a result, accurate concentration estimation was successfully performed.

\section{Introduction}

The deterioration of the environment caused by bad smells and volatile organic compounds has become one of the problems in our daily life. A rapid and low-cost sensing system is thus indispensable for monitoring of the environment. Although gas chromatography/mass spectrometry (GC/MS) is the most popular method, it is expensive, time-consuming and not portable. Moreover, GC/MS requires skilled operators. A semiconductor gas sensor and an electrochemical gas sensor can also be used but their selectivities are relatively broad.

In this study, we focused on the gas detector tube method, ${ }^{(1)}$ a well-known simple method for gas measurement. Although manual reading of the discoloration-layer

*Corresponding author: e-mail: nakamoto@mn.ee.titech.ac.jp 
length in the gas detector tube is typical, reading errors cannot be ignored because the discoloration boundary is often ambiguous. Moreover, manual reading requires labor and lacks accuracy. Thus, we developed a system to measure the color change of the gas detector tube automatically and continuously using a one-dimensional CCD sensor and a mobile phone with a two-dimensional image sensor. ${ }^{(2-4)}$ The distribution of bad smell concentration around a factory has been measured using multiple sensor nodes. ${ }^{(5)}$ However, it is impossible to read many gas detector tubes at the same time using a onedimensional CCD sensor. Using the mobile phone camera, we cannot calculate the gas concentration inside the mobile phone images although images can be sent to a host computer. However, it is very costly to send image files to the host many times. It is better to calculate the concentration within the measurement system from the viewpoints of cost and time required for communication.

Thus, we aimed to develop a new sensing system by combining the gas detector tube with a built-in camera module and a CPU core embedded into a field programmable gate array (FPGA). The system we developed can read many gas detector tubes simultaneously and the embedded CPU calculates the concentration using image data directly from the built-in camera module. ${ }^{(6)}$

Although the measurement was automated using our system, another problem was revealed. Because digital cameras are sensitive to color changes, a slight change due to humidity, which is not a problem in manual inspections, cannot be ignored in our system. There are several methods to remove humidity. However, the sample gas is removed together with the humidity in some cases, and it is particularly difficult to separate a sulfide compound such as hydrogen sulfide from humidity. Thus, a humidity sensor was added to the system. However, simple humidity compensation methods such as linear regression did not work because humidity affected the data in a complicated manner. Therefore, a neural network was used for humidity compensation. We propose the humidity compensation method and present experimental results in this paper.

\section{Experimental Setup}

A gas detector tube is a device that shows a color change due to gas exposure. When it is exposed to a gas, the reagent in the detector tube reacts to the gas and its color changes, as shown in Fig. 1. The sample gas was extracted by an air pump at a flow rate of $60 \mathrm{~mL} / \mathrm{min}$. The length of the discoloration layer indicates the gas concentration.

A block diagram of the measurement system is shown in Fig. 2. The system consists of a camera module (Akitsukidenshi MTV-54K0DN, $858 \times 525$ pixels), an FPGA, a static RAM (SRAM), an air pump, a light source and a host computer.

The camera has a digital output as well as an analog output (communication interface, ITU-656; data format, YUV422). The control logic and CPU core (Nios3.2, Altera) were implemented into the FPGA (Altera, EP1C12Q240C8). The circuits were described in VHDL language.

Images of detector tubes taken by the camera module are stored in four SRAMs (IDT; IDT71V424S12PH; $512 \mathrm{k} \times 8$ bits, access time, $12 \mathrm{~ns}$ ). Two SRAMs are used for a reference image and the other two are used for a measured image. The difference 


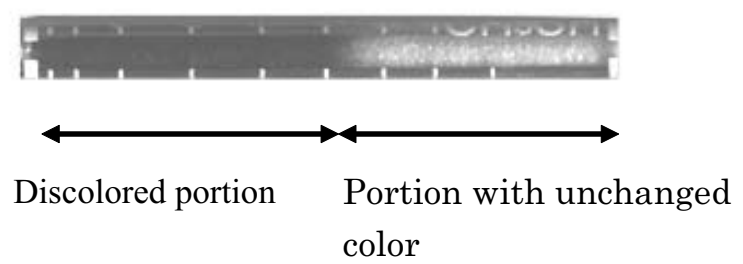

Fig. 1. Photograph of discoloration in gas detector tube owing to vapor exposure (sample gas, methyl mercaptan).

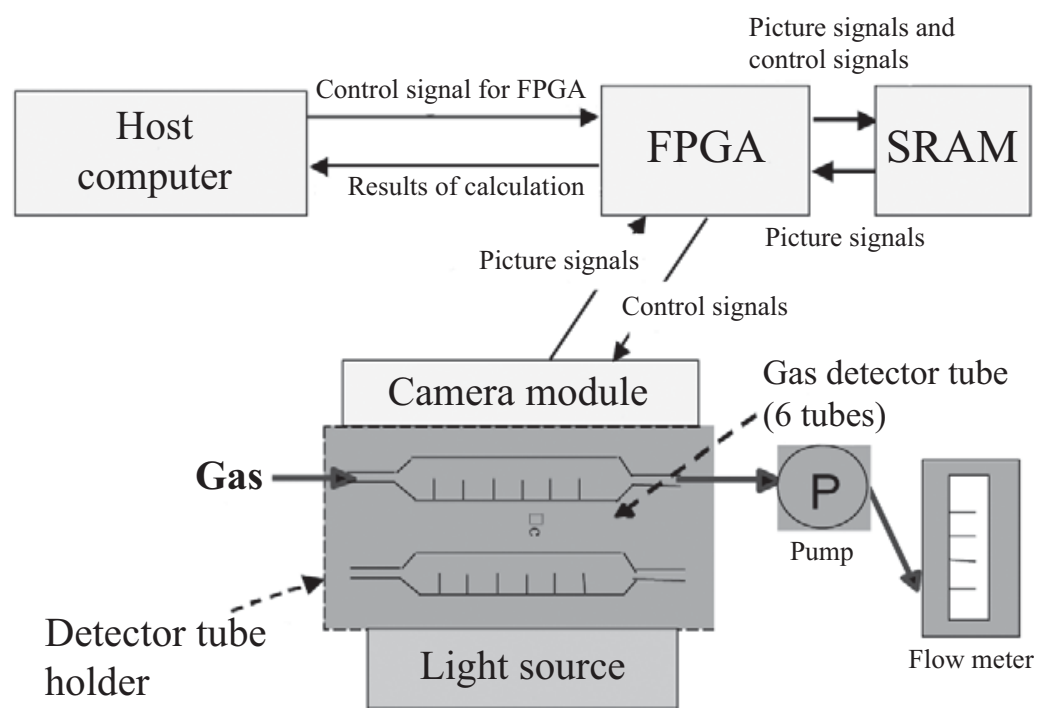

Fig. 2. Entire structure of bad-smell sensing system.

between the two images is used for gas concentration estimation. Image data are directly transferred from the camera module to the SRAMs without using the CPU core because the image transfer speed is too high for a CPU core. Then, the CPU core reads the image data stored in the SRAMs and calculates the discoloration speed. The program of the CPU core was written in $\mathrm{C}$ language. The discoloration-area data are transferred to the host computer via a serial port.

We have developed software (Visual Basic 6.0) for the host computer to control the FPGA circuit. Measurement parameters such as the number of images and the measurement interval can be input using this software, and the host computer sends these parameters to the CPU core in the FPGA. After a user simply sets up the gas detector tubes and sets these parameters, the system we developed automatically measures the gas concentration after the operator clicks the start button. It takes $90 \mathrm{~s}$ to send an image of one detector tube from the FPGA to the host computer via an RS232C 
interface. However, the time of transmission is less than $1 \mathrm{~ms}$ when only information on discoloration speed is sent.

It is necessary to set the detector tubes and light source in a box to eliminate ambient light. However, it is not easy to illuminate the detector tubes uniformly to obtain their stable images. The problem is caused by shadows or reflection from the cylindrical glass surface of the detector tubes. To overcome this problem, the transmission light through the gas detector tubes is detected using a camera module. In the system we developed, up to six gas detector tubes can be simultaneously used to monitor the concentrations of multiple gases. However, only one gas detector tube was used here for simplicity.

\section{Method of Calculating Discolation-Layer Area}

Figure 3 shows images taken by our developed system. Figure 3(a) shows the reference image taken before discoloration and Fig. 3(b) shows the discoloration image after gas exposure. Figure 4 shows the difference between the two images, which is used to calculate the discoloration-layer area as described below. In Fig. 4, the blue zone on the left side is the brightness change of the gas detector tube exposed to hydrogen sulfide.

Let us consider $S$ as the sum of the brightness changes of the gas detector tube and $P$ as the number of particles discolored owing to the chemical reaction between all the particles in the gas detector tube and the analyte. The relationship between $S$ and $P$ is given by

$$
S \propto P .
$$

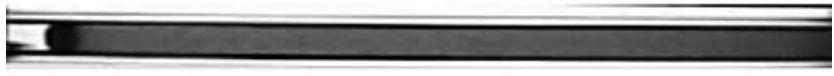

(a)

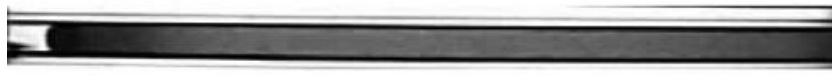

(b)

Fig. 3. Images taken by developed system (sample gas, hydrogen sulfide). (a) Before vapor exposure and (b) after vapor exposure (sample gas, hydrogen sulfide).

Fig. 4. Difference between the two images in Fig. 3. 
Figure 5 shows an example of the discoloration layer in the gas detector tube. $f_{\mathrm{ya}}$ is the sum of brightness changes along the $\mathrm{Y}$ axis after discoloration. $\Delta x$ is the sampling interval of captured images. The discoloration-layer area $S$ is defined as

$$
S=\sum_{i=1}^{n} f\left(x_{i}\right) \Delta x
$$

where $n$ is the number of sampling data, $x_{i}$ the distance of the $i$ th pixel from the origin and $f\left(x_{i}\right)$ the sum of brightness changes along the $\mathrm{Y}$ axis at position $x_{i}$.

Figure 6 shows the relationship between time and $S$ during the exposure to hydrogen sulfide. It can be noted from Fig. 6 that the slope increases as gas concentration

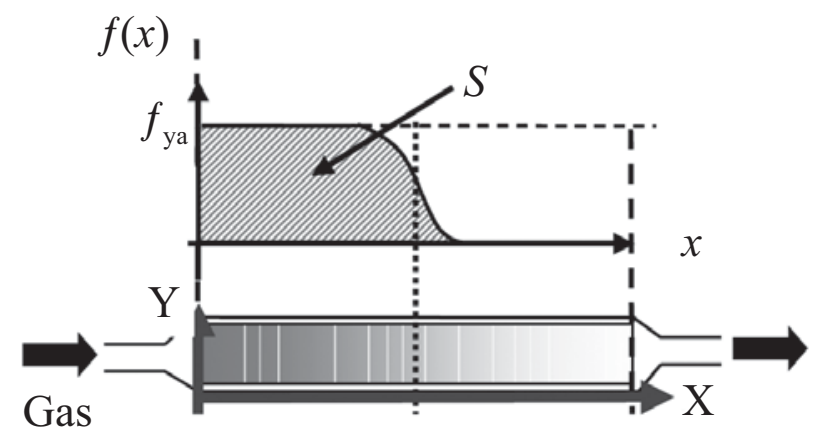

Fig. 5. Method of calculating discoloration-layer area $S$.

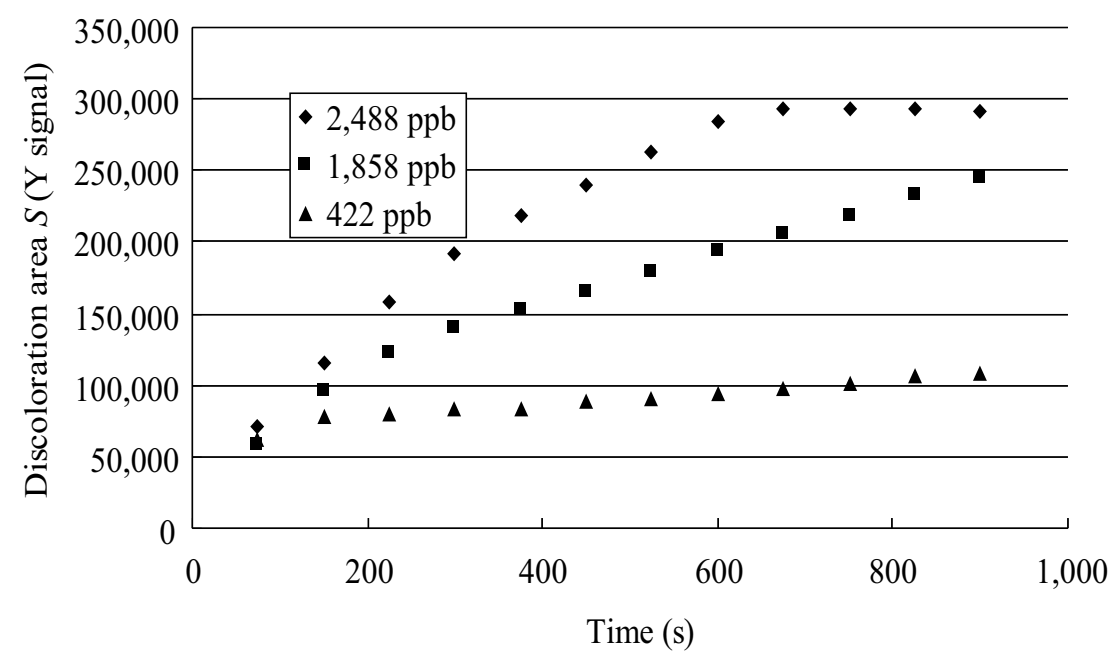

Fig. 6. Relationship between time and $S$ as a parameter of concentration (sample gas, hydrogen sulfide). 
increases. We define this slope as the discoloration rate, which is dependent on gas concentration. The discoloration rate is obtained using the least-squares method. We can make a calibration curve between concentration and discoloration rate for each type of gas. Then, the gas concentration can be calculated using the calibration curve and discoloration rate. The curve for a concentration of 2,488 ppb in Fig. 6 saturates at $600 \mathrm{~s}$ because all particles have changed their color. Therefore, only the unsaturated part is used to calculate the discoloration rate.

\section{Effect of Humidity}

We confirmed the influence of humidity, although it is not a problem in the case of manual inspection. There are two portions in the gas detector tube, namely, a discolored portion and a portion with unchanged color, as shown in Fig. 1. We separately discuss these two portions. We used a gas detector tube ${ }^{(7)}$ for hydrogen sulfide (No. 4LL, Gastec) as described in the following section.

\subsection{Response to humidity in portion with unchanged color}

Although the color change in this portion is subtle in the direct visual inspection, its brightness change is clear in a captured image. The brightness change due to humidity is shown in Fig. 7. It can be seen from the figure that the influence of humidity appears after that of the target gas. The response to humidity becomes saturated a few minutes after the beginning of gas exposure.

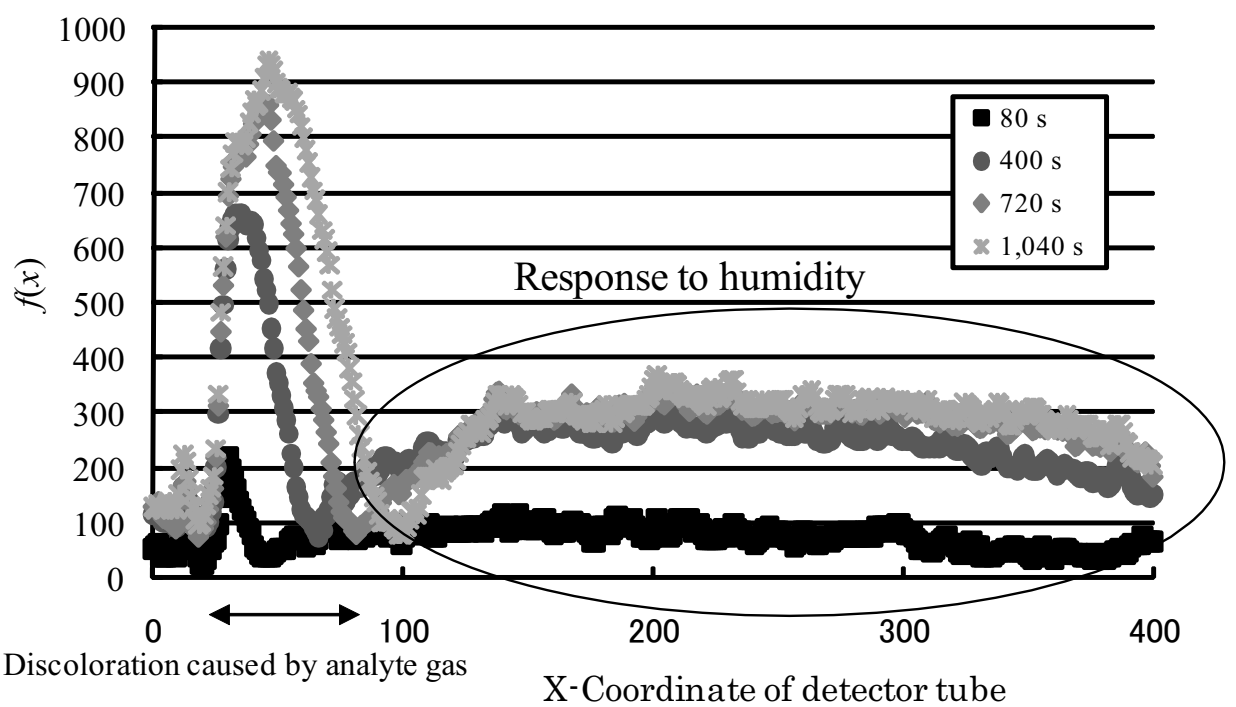

Fig. 7. Example of effect of humidity in portion of unchanged color (Y signal; hydrogen sulfide, $594 \mathrm{ppb})$. 
As a result of the response to humidity, the discoloration rate at an earlier stage is higher than that after $400 \mathrm{~s}$ as shown in Fig. 8. Two methods were proposed to suppress the effect of humidity. In the first method, the brightness change below a threshold at each pixel can be regarded as zero in the calculation of $S$. The results of the threshold method are shown in Figs. 9 and 10. It can be seen from Fig. 9 that the response to

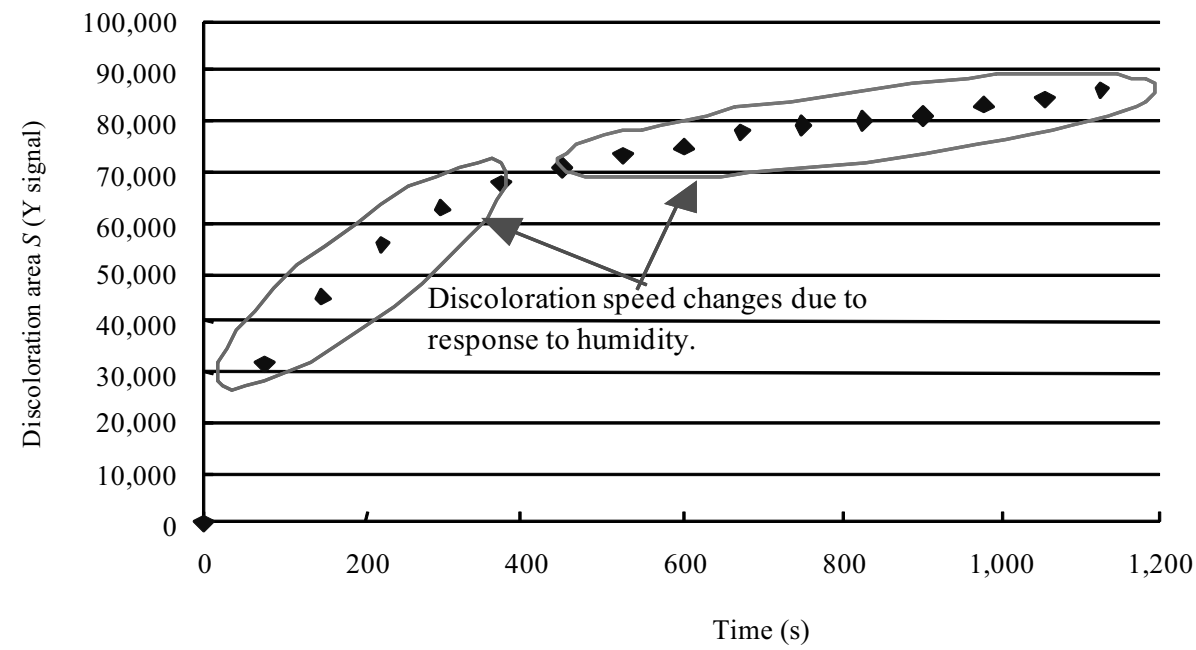

Fig. 8. Relationship between time and $S$ when humidity affects portion with unchanged color (Y signal; hydrogen sulfide, $594 \mathrm{ppb}$ ).

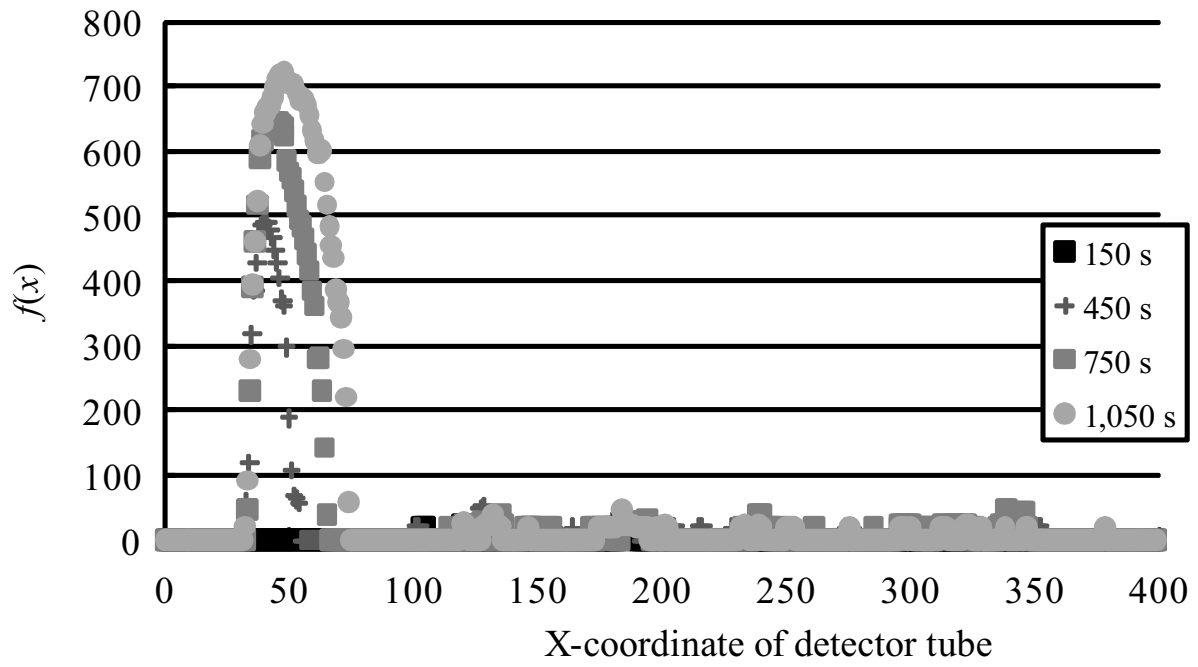

Fig. 9. Relationship between distance and brightness change when threshold method is applied (Y signal; threshold, 18; hydrogen sulfide, $594 \mathrm{ppb}$ ). 


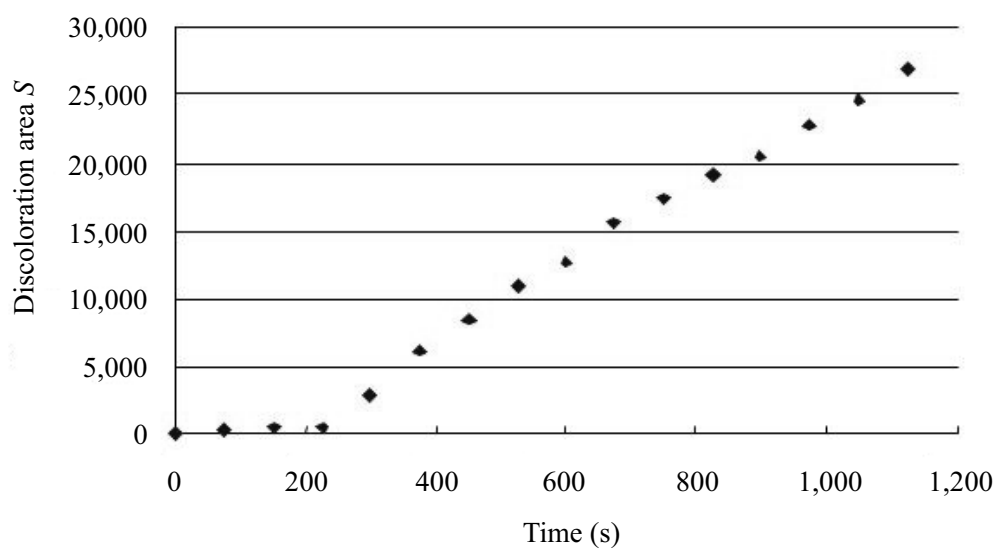

Fig. 10. Relationship between time and $S$ when threshold method is applied (Y signal; threshold, 18; hydrogen sulfide, $594 \mathrm{ppb}$ ).

humidity in the portion with unchanged color was suppressed. Thus, the sudden increase in the discoloration rate between 0 and $80 \mathrm{~s}$ in Fig. 8 was not observed in Fig. 10. $S$ was zero before $200 \mathrm{~s}$ because the brightness change due to gas exposure was below the threshold.

Another method to suppress the effect of humidity in the portion with unchanged color is as follows. The gas detector tube is exposed to ambient air for a sufficient time in advance (empirically $15 \mathrm{~min}$ ) to saturate the response to humidity before the actual measurement. The sample gas is then introduced into the gas detector tube. The results are shown in Figs. 11 and 12. The effect of humidity can be suppressed in the way similar to the threshold method. The sudden increase in $S$ immediately after the start of measurement was not observed. Moreover, the data immediately after the start of measurement can be used to calculate the discoloration rate, whereas these data cannot be used in the threshold method. Using either of the two methods, the response to humidity in the portion with unchanged color can be canceled.

\subsection{Dependence of humidity on response in discolored portion}

A photograph of the gas detector tubes exposed to a gas of the same concentration under different humidity levels is shown in Fig. 13. The figure revealed that the color due to discoloration depended on humidity despite the same discoloration length. The discoloration length can be used to determine the concentration when the gas concentration is high enough to obtain a clear boundary between the discoloration portion and the unchanged portion. However, this boundary is ambiguous in lowconcentration vapor and the image depends on the humidity even if the concentration is the same. Although we aim to use this method in this complicated situation in the near future, a gas with a moderate concentration was studied at the current stage. 


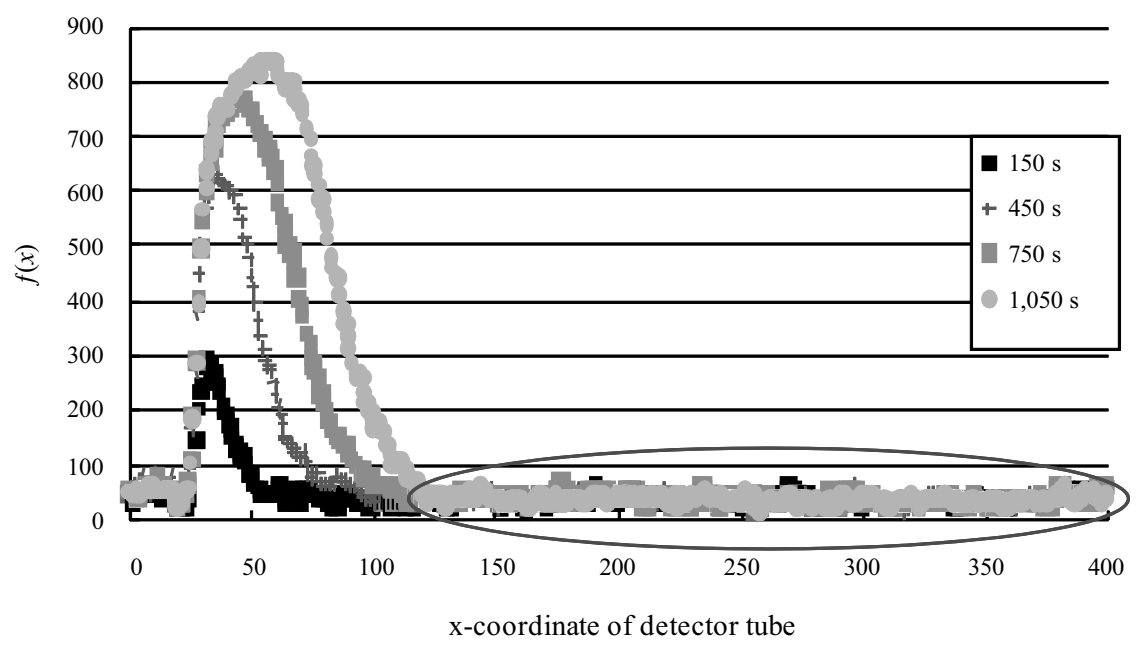

Fig. 11. Relationship between distance and brightness when detector tube is exposed to ambient air in advance (Y signal; hydrogen sulfide, $594 \mathrm{ppb}$ ).

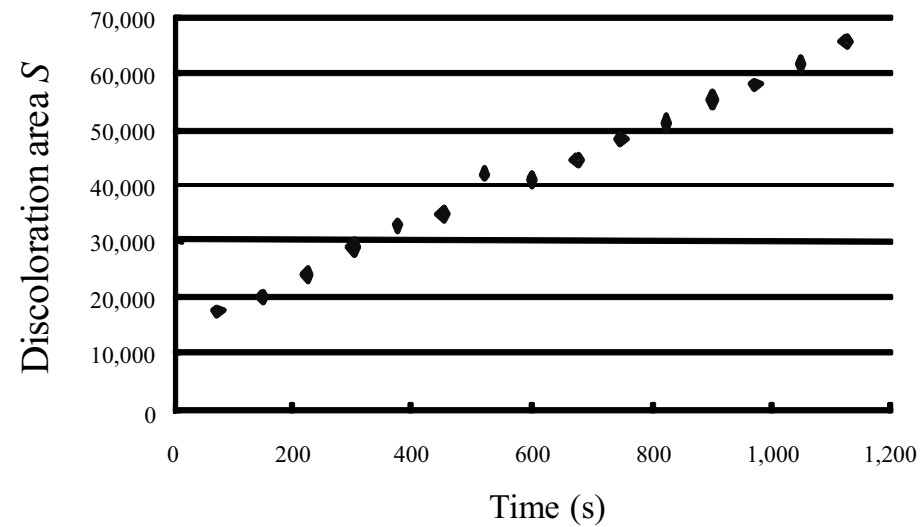

Fig. 12. Relationship between time and $S$ when gas detector tube is exposed to ambient air in advance (Y signal; hydrogen sulfide, $594 \mathrm{ppb}$ ).

Then, the humidity sensor (SHT75, Sensirion) was implemented into a measurement system with a digital output with a serial interface. The CPU core in the FPGA reads the data from the humidity sensor and transfers the humidity data together with the discoloration-area data to the host computer. The humidity sensor was placed prior to the gas detector tube. 


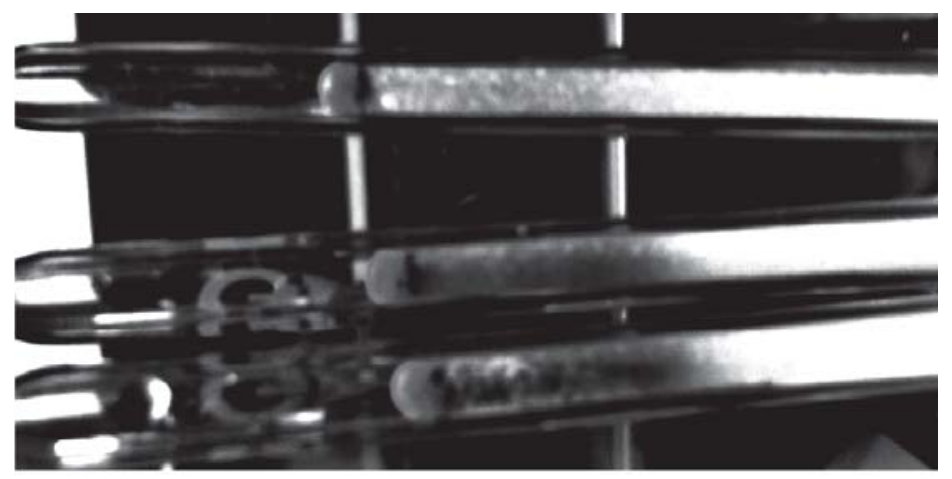

Fig. 13. Dependence of discoloration-layer color on humidity. Gas, $700 \mathrm{ppb}$ hydrogen sulfide. Humidities, $8.5 \%$ for top tube, $75 \%$ for middle tube, $96 \%$ for bottom tube.

\subsection{Method of sample gas preparation}

In the case of compensating data using the humidity sensor, a gas of the same concentration at different humidity levels should be prepared during the calibration. In this study, a sampling bag was adopted as shown in Figs. 14 and 15. An aqueous solution of hydrogen sulfide was placed into a fluorine-containing resin bag (GL Science, $20 \mathrm{~L}$ ) using a microsyringe. Then, humidified air was packed into the sampling bag, as shown in Fig. 14. Humidity can be controlled by adjusting the flow rates of two mass flow controllers (MFCs), as shown in Fig. 15. The aqueous solution was completely evaporated using a heater. Fine adjustment of the gas concentration was performed by concentration monitoring using a photoionization detector (PID, PGM-7240, RAE Systems).

\subsection{Experimental results}

We investigated the response of the gas detector tube to hydrogen sulfide at different humidity levels. The gas detector tube was exposed to the ambient air in advance in the same manner as that in $\S 4.1$. The results are shown in Figs. 16 and 17. It can be seen from Fig. 16 that the relationship between humidity and discoloration rate changes as a parameter of gas concentration. Figure 17 shows the relationship between gas concentration and discoloration rate. In both figures, simple linear regression is inappropriate since there is a complicated relationship among the discoloration rate, gas concentration and humidity.

\section{Humidity Compensation by Neural Network}

Because the mapping of discoloration rate and humidity to gas concentration is not simple, we used neural network. Arbitrary mapping can be approximately performed using a multilayer perceptron (MLP) neural network. The mapping can be carried out 




Fig. 14. Preparation of sample gas using sampling bag.



Fig. 15. Setup for humidity adjustment.



Fig. 16. Relationship between humidity and discoloration rate as a parameter of gas concentration. 


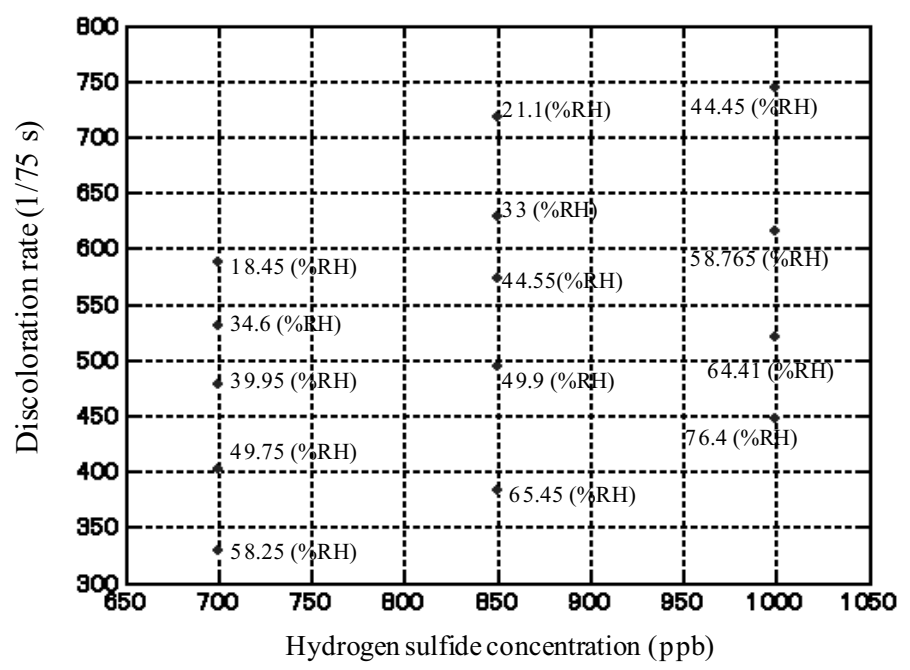

Fig. 17. Relationship between hydrogen sulfide concentration and discoloration rate as a parameter of humidity.

even when a concrete mathematical model does not exist. It works on the platform of MATLAB (Mathworks Inc.). The input signals were discoloration rate and humidity. The output signal was the gas concentration after humidity compensation. After the discoloration rate $(1 / 75 \mathrm{~s})$ and concentration $(\mathrm{ppb})$ were divided by 10,000 and the humidity $(\% \mathrm{RH})$ was divided by 100 , these data were input into the MLP neural network. The MLP neural network was trained using the back-propagation algorithm, ${ }^{(8)}$ and the concentration estimation was validated by the leave-one-out method. ${ }^{(9)}$

We optimized the number of neurons in the intermediate layer, the number of trainings and the learning rate. The sum of the squares of errors between the estimated and true concentrations became smallest when the number of neurons in the intermediate layer was 8 , the number of trainings was 20,000 and the learning rate was 0.25 .

The result of gas concentration estimation using the leave-one-out method is shown in Fig. 18. Because the estimated concentration is highly correlated with the actual concentration, the compensation of the effect of humidity using the neural network was successfully performed.

Then, we attemted to compensate for changes in humidity using a self-organized map (SOM). ${ }^{(10)}$ Although a SOM is originally unsupervised, it can also be used for mapping. It is expected to compensate for changes in humidity similarly to mapping using the MLP neural network. Moreover, it is easier to implement a SOM into LSI because of its simple structure. It is excellent for compensating for the humidity change using a chip embedded into the sensing system. Thus, a preliminary experiment on humidity compensation using a SOM before hardware implementation was performed here. 


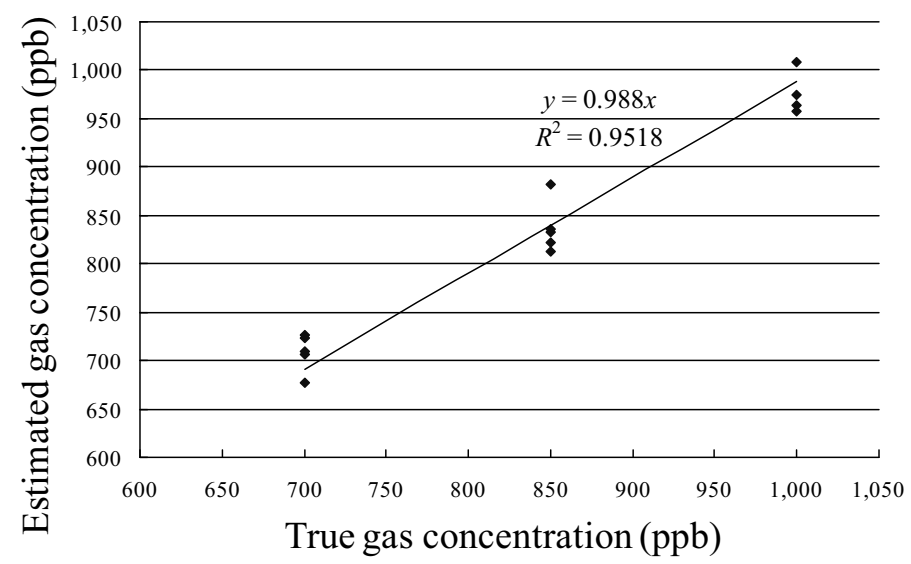

Fig. 18. Relationship between true concentration and estimated concentration under varying humidity when an MLP neural network is used (sample gas, hydrogen sulfide).

When the dimension of the data vector is $m$ and the number of outputs mapped from the input space is $n$, an $n+m$-dimensional vector is used as the reference vector. The SOM is trained using these vectors during the learning process. In the estimation process, the reference vector closest to the input vector is selected. Distance is calculated only using the $m$-dimensional portion corresponding to the data vector. Then, the $n$-dimensional portion of the closest reference vector is the corresponding estimated output. $m$ is two (humidity and discoloration speed) and $n$ is one (estimated concentration) in the present study.

The map obtained after training is shown in Fig. 19. The map is laterally divided by concentration. The estimation result using the leave-one-out method is shown in Fig. 20. The map size was $10 \times 10$ and the initial radius of the neighbor was 1 . It was found from the figure that the concentration estimation was successful using a SOM.

\section{Conclusions}

We have developed an automated bad-smell sensing system using gas detector tubes and a built-in camera. The problem of the effect of humidity was apparent in the automated measurement of the gas detector tube, whereas humidity is not a problem in the manual inspection of a gas detector tube.

We overcome this problem by introducing a humidity sensor and a neural network into the system. Because neural networks such as an MLP neural network and a SOM enable an arbitrary nonlinear mapping, compensation for the effect of humidity was successfully achieved. 




Fig. 19. Self-organized map for various concentrations under varying humidity (number of trainings, 100; map size, $7 \times 7$; radius of neighbor, 1).



Fig. 20. Relationship between true concentration and estimated concentration at various humidities when a SOM is used. Sample gas, hydrogen sulfide.

In future, we plan to measure the vapors with concentrations of a few tens of ppb because the concentration used in this study was relatively high. Since it is possible to detect a gas with a concentration of a few tens of $\mathrm{ppb},{ }^{(3)}$ the concentration level in this study can be further reduced. Moreover, the measurement system becomes more useful when humidity compensation can be carried out in real time in the measurement system. 


\section{Acknowledgment}

We wish to thank Mr. Junichi Ide of T. Hasegawa Co., Ltd. for providing samples.

\section{References}

1 W. Beather: Sensor Updates 4 (1998) 82.

2 Y. Tanaka, M. Yoshioka, T. Nakamoto and T. Moriizumi: Trans. IEEJ 124-E (2004) 321 (in Japanese).

3 Y. Tanaka, T. Nakamoto and T. Moriizumi: Sens. Actuators, B 119 (2006) 84.

4 H. P. Ninh, Y. Tanaka and T. Nakamoto: Sens. Actuators, B 125 (2007) 138.

5 T. Nakamoto, Y. Tanaka and H. P. Ninh: IEEJ Trans. SM 127 (2007) 359.

6 T. Ikeda, H. Hirano, T. Nakamoto and T. Arimoto: Proc. IEEJ Sensor Symp. 2008 (Okinawa, Japan, 2008) p. 159.

7 Environmental Analysis Technology, 5th Edition (Gastech, Japan, 2004).

8 D. E. Rumelhart, J. L. McClelland and PDP Research Group: Parallel Distributed Processing (MIT Press, USA, 1986).

9 R. O. Duda, P. E. Hart and D. G. Stork: Pattern Classification (Wiley-Interscience, USA, 2001).

10 T. Kohonen: Self-Organization and Associative Memory (Springer-Verlag, Germany, 1987) pp. 119-157. 